\title{
Abstracts for a student research week for March 2021
}

The abstracts in this supplement were presented during student research week at Texas Tech University Health Sciences Center in Lubbock in March 2021. The students involved in these projects participated in the 2020 summer research program. The director of this program is Leslie Shen PhD at Lesie.shen@ttuhsc.edu.

\section{Jonathan Abraham}

\section{Ponseti Casting and Soft Tissue Release for the Initial Treatment of Non-Idiopathic Clubfoot}

Jonathan Abraham, Jon Cooper Wall Jr, Cody Beaver, Michel Diab

Ponseti casting has universally been accepted as the gold standard for treatment of idiopathic clubfoot. Conversely, primary treatment for non-idiopathic clubfoot has not been established. Traditionally, these feet have been treated with primary soft tissue release (STR) and serial casting. However, this approach has led to variable success often times requiring secondary surgery. Recently, the Ponseti method has been used to treat non-idiopathic clubfoot and mid-term results from several institutions have been promising. The purpose of this study is to compare the treatment outcomes following primary STR and Ponseti casting.

An IRB-approved retrospective study of patients treated for non-idiopathic clubfoot between 2005 and 2020 was conducted. Patients were included if they began treatment before the age of 2 and had at least one year of follow up. Patients were placed into either the STR group or Ponseti group and variables of interest were documented. Data was analyzed using Mann-Whitney $U$ test for continuous variables.

A total of 33 children with 57 neuromuscular/syndromic clubfoot were identified of which 9 (15 feet) were treated with STR and 24 (42 feet) were treated with Ponseti casting. Patients treated with STR were found to have significantly more surgeries performed over the course of treatment than those treated with Ponseti casting $(p=.001)$ with an average of 4.2

Corresponding author: Leslie Shen Contact Information: Leslie.shen@ttuhsc.edu DOI: $10.12746 /$ swrccc.v9i38.831 surgeries in the STR group and 1.5 surgeries in the Ponseti group. Extracapsular procedures were performed in $100 \%$ of the STR group and $97.6 \%$ of the Ponseti group $(p=.55)$. Intracapsular procedures were performed in $100 \%$ of the STR group and $50 \%$ of the Ponseti group $(p=.001)$.

The Ponseti method should serve as the primary approach in the initial treatment of non-idiopathic clubfoot as it can reduce the risk of future invasive intracapsular surgery, decrease complication rate, and shorten anesthesia and surgery times when surgical treatment is necessary.

\section{Veena Agusala}

\section{CHF outcomes in Ethnic groups: Do they differ?}

Veena Agusala, Nandini Nair

Considering established social and ethnicallybased determinants of health, African-American and Hispanic congestive heart failure patients will display overall worse outcomes post-intervention with greater risk of developing hypertension, diabetes, COPD, and renal insufficiency.

By 2017, CHF was a contributing cause to 1 in 8 deaths according to the $\mathrm{CDC}^{4}$. In addition, racial disparities continue to pose a major issue in healthcare. While the incidence of $\mathrm{CHF}$ is equally frequent in men and women, African-Americans are 1.5 times more likely to develop heart failure than Caucasians ${ }^{2}$.

By recording ethnicity as well as outcome variables commonly associated with CHF patient health, we hope to find possible areas of care to improve.

We utilized a standard chart review for data collection from charts of all patients of each ethnic group: African-American, non-Hispanic White, Hispanic, all with a history of CHF intervention at UMC in the last 2 years. The data points collected included survival, 
ejection fraction, BMI, age, gender, renal function, and comorbidities. We characterized outcomes based on the development of significant comorbidities: hypertension, diabetes, COPD, and renal insufficiency.

The study population was $46.8 \%$ female and $53.2 \%$ male, with a mean BMI of 31.1 (obese).

As hypothesized, Hispanic patients were most likely to have diabetes already before intervention $(p=.001)$, to develop diabetes post intervention $(p=.001)$, and to develop hypertension post discharge $(p=.008)$. In contrast, non-Hispanic White patients were most likely to have COPD before intervention $(p=.001)$, and to develop COPD after CHF discharge $(p=.001)$.

UMC patient data reflects racial and ethnic disparities in patients post-CHF intervention, in addition to a dangerously elevated average BMI. Racial and ethnic differences in risk of developing comorbidities postintervention must be considered in treatment.

\section{Kiran Ali}

\section{COVID-19 Hospitalized Patients' Demographic Parameters and Outcomes}

Kiran Ali, Sanjana Rao, Jeff Dennis, Gilbert Berdine, Victor Test, Kenneth Nugent, Haneen Mallah

The severity of COVID-19 ranges from asymptomatic subclinical infections to severe acute respiratory failure requiring mechanical ventilation. Patients admitted to the hospital have increased mortality rates and patients requiring intensive care have significantly increased mortality rates. Multiple factors influence these outcomes. This study used simple demographic information available on admission to evaluate possible associations between these variables and outcomes, including mortality and length of stay. Clinical outcomes in 63 patients admitted to a tertiary care hospital in West Texas were reviewed. Older patients, patients admitted from nursing homes, and patients admitted to medical intensive care units had increased mortality. Unadjusted analysis indicated that males had increased mortality. Adjusted analysis indicated that males spent nearly 5 days longer in the hospital than females. In summary, age, chronic illness requiring nursing home placement, and acute severe illness requiring intensive care unit admission identify patients with worse prognoses. In addition, males will likely have a longer length of hospital stay.

\section{Emma Brackett}

\section{Characterization of Amino Acid Substitutions in the Binding Site of Bupropion in GLIC}

Dubem Onyejegbu, Jessica Shepherd, Elham

Pirayesh, Akash Pandhare, Zackary R. Gallardo, Michaela Jansen, Emma Brackett

The Gleobacter ligand-gated ion channel (GLIC) is a pentameric ligand gated ion channel ( $\mathrm{pLGIC}$ ) that is a homolog to the eukaryotic Cys-loop receptor superfamily. Cys-loop eukaryotic ion channels include serotonin receptor, GABA receptor, and nicotinic acetylcholine receptor. GLIC, a cationic selective ion channel, has been used to investigate structure and function of eukaryotic pLGICs. GLIC is proton activated channel that contains an extracellular and transmembrane domain. Bupropion, an atypical antidepressant, and smoking cessation drug has been shown to non-competitively inhibit pLGICs. For therapeutic use, bupropion blocks the reuptake of dopamine and norepinephrine in the brain. GLIC mutants were used to analyze and determine the binding site and important residues in the ion channel for bupropion to better understand the mechanism of action of the atypical antidepressant. A construct was formed by site-directed mutagenesis of the transmembrane domain of GLIC with the intent to impede the binding of bupropion to the channel. The construct was injected and expressed in Xenopus oocytes. Twoelectrode Voltage Clamp was used to calculate the $\mathrm{pH} \_50$, which is the proton concentration needed to cause half of maximal activation of GLIC mutants. Following the determination of $\mathrm{pH} \_50$, bupropion was added to the solution to determine its ability to bind and inhibit the mutated channel. Cysteine scanning was preformed to identify key residues in the binding site of bupropion to GLIC. Further studies need to be done to compare the binding site on GLIC to its eukaryotic homologs to better understand bupropion's mechanism of action of so its therapeutic uses are well understood and can be adjusted accordingly. 


\section{Whitney Brantley}

\section{Effect of BMI on large bore chest tube vs. pigtail catheter}

\section{Logan Warren, Whitney Brantley, Samudani Dhanasekara, Robyn Richmond}

Pigtail catheters (PCs) and large bore chest tubes (LBCTs) have been found to have similar efficacy rates in patients with pneumothorax (PTX), hemotho$\operatorname{rax}(\mathrm{HTX})$, or hemopneumothorax (HPTX). However, literature exploring factors that contribute to failures in tube thoracostomy is limited. The purpose of our study was to compare the BMI and the failure rates of PCs and LBCTs in traumatic PTX, HTX, and HPTX. We hypothesized that an increased BMI directly correlates to an increased risk of PC complication and failure rate. A Level 1 trauma center registry was queried to include all trauma patients that underwent $\mathrm{PC}$ or LBCT thoracostomy between June 2015-April 2020. Clinical outcomes of the two groups were compared using independent sample t-tests and Chi square tests. To evaluate outcomes in relation to BMI, each variable was regressed on $\mathrm{BMI}$ in a series of univariate logistic regression models and multiple logistic regression models that included the type of intercostal tube as a categorical covariate performed using R. Out of 183 patients, 54 had PC and 129 had LBCT. There was no significant difference in demographic parameters between the two groups except BMI, which was significantly high in the LBCT group. BMI significantly predicted an increase in $30 \mathrm{~d}$-morbidity and mortality in univariate logistic regression models. These associations remained significant after controlling for the type of intercostal tube. However, BMI failed to significantly predict all other examined variables including tube failure, post-tube PTX and readmission rates. Our hypothesis that an increased BMI directly correlates to an increased risk of PC complication and failure rate did not hold true based on our findings. However, our findings indicated that with increasing BMI, there is an increment of 30d-morbidity and mortality related with intercostal tube placement. As obesity is rising in the US, surgeons must prepare to anticipate complications related with either PC or LBCT placement.

\section{Elizabeth Brown}

\section{Curriculum mapping to facilitate curricular renewal: The Immune System}

Elizabeth Brown, Emily Sargent, Abigail Jackson, Michaela Jansen

The medical school curriculum at TTUHSC School of Medicine consists of four years with the first two years constituting the basic sciences or preclerkship curriculum and the last two years constituting the core clerkships and additional clinical training. Here we mapped the curriculum content for the preclerkship curriculum. At present the SOM is embarking on a journey to renew the curriculum. The current curriculum begins with two general foundational courses (Clinically Oriented Anatomy and Biochemistry, Cells and Tissues) and then enters a curriculum that is a combination of discipline and organ-system-based curriculum. Students are first introduced to the normal physiological function of the major organ systems during year one and then revisit these systems for coverage of the pathophysiology and treatment of disease during year two. The SOM curriculum revision will continue to start with Anatomy and General Principles and then proceed in a strictly organsystem based manner combining physiology and pathophysiology for each organ system while integrating relevant concepts of biochemistry, cell biology and histology. We used the United States Medical Licensing Examination (USMLE) Step 1 content outline and mapped the content lists against the detailed contents of the blocks. The curriculum mapping facilitated the identification of gaps and redundancies, as well as the relative quantification of instructional methods used. For the immune system, we found that development and regulation of the adaptive immune response, immunoglobulins, and specific immunodeficiencies were overrepresented. However other topics were underrepresented such as termination of the immune response, certain biologically active antibodies, vaccine adverse effects, age and immunity, and some specific drugs effects. Our detailed curriculum inventory provides an essential framework to optimize and refine content representation and distribution during the current curricular renewal phase. 


\section{Angela Chen}

\section{Foix-Chavany-Marie Syndrome due to Unilateral Anterior Opercular Infarction with Leukoaraiosis}

Katherine Rivas, Jie Pan, Angela Chen, Bailey

Gutierrez, Parunyou Julayanont

FCMS is a cortical-subcortical pseudobulbar palsy characterized by impairment of facio-masticatorypharyngo-glosso-laryngeal voluntary movement with preservation of automatic and involuntary movements. FCMS is typically caused by vascular insults on the bilateral anterior opercular or adjacent subcortical areas. Acute onset of FCMS secondary to a unilateral lesion is extremely rare. An 83-year-old right-handed woman presented with sudden onset of severe dysarthria, dysphagia and left facio-brachio-crural weakness. Neurological examination revealed severe spastic dysarthria, left upper motor neuron type of facial paralysis and left-sided hemiparesis. Emotional facial movement was intact with disturbed volitional facial movement. She had no aphasia, alexia or agraphia. A swallowing evaluation confirmed severe oropharyngeal dysphagia. MRI brain showed acute infarction on the right frontal operculum and preexisting extensive bilateral leukoaraiosis without evidence of brainstem lesion. During the two-week follow-up her symptoms had partially improved. An acute unilateral anterior opercular lesion can decompensate preexisting bilateral or contralateral corticobulbar-subcortical lesions and can result in the typical features of FCMS. This case demonstrates a favorable recovery of FCMS secondary to a unilateral lesion compared to bilateral lesions.

\section{Kevin Chin}

\section{Post-Intervention Effects of Tai Chi and Qi Gong on Depression Scores: A Systematic Review and Meta-Analysis}

Kevin Chin, Haven Ward, Chathurika Dhanasekara, Dale Dunn, Chwan-Li Shen, Chanaka Kahathuduwa

Background: Tai Chi and Qi Gong are mind-body interventions with positive effect on psychological well-being. The purpose of this study was to establish the beneficial effects of exposure to at least 3 weeks of Tai Chi or Qi Gong on depressive scores in a systematic review and meta-analysis.

Methods: Peer-reviewed studies covering the effects of Tai Chi and Qi Gong on depressive or anxiety symptoms were searched on PubMed, Medline, ProQuest, Scopus, and Web-Of-Science databases. A screening process was implemented, which included two independent researchers assessing the studies for eligibility. Full text articles of prospective controlled trials examining the effects of $>3$ weeks of Tai Chi or Qi Gong on depressive symptoms were identified. Studies were evaluated for risk of bias per the Cochrane handbook. Pre-and post-intervention depressive symptoms of intervention and control groups were extracted. DerSimonian Liard randomeffects meta-analyses were performed using the meta package in $R$ (4.0.3).

Results: Out of the 1,306 studies that emerged upon an initial database search, 74 studies met eligibility (mean exposure $31.53 \pm 37.10$ hours; range 5-312). Tai-Chi training significantly decreased standardized depression scores compared to the controls (Cohen's $\mathrm{d}=-0.30,[-0.42,-0.17])$. Similarly, Qi Gong training also significantly decreased standardized depression scores vs. controls (Cohen's $d=-0.39,[-0.51,-0.26]$ ).

Discussion: Exercises which focus on mindfulness such as Tai Chi and Qi Gong seem to have a moderate effect in alleviating depression. Our findings should promote increased recommendation of these mind-body interventions to reduce clinical as well as subclinical depression. Future meta-regression analyses should examine the differential effects of mind-body interventions based on socioeconomic factors and symptom severity. Furthermore, future randomized trials should compare the effectiveness of these interventions vs. anti-depressive medications.

\section{Daniel De Simon}

\section{Evaluation of surgical outcomes of neonates undergoing surgery during their initial hospital stay in the level 4 Neonatal Intensive Care Unit (NICU) at Covenant Children's Hospital (CCH)}

Daniel De Simon, Celeste Hollands

The purpose of this study is to evaluate surgical outcomes of neonates undergoing surgery 
during their initial hospital stay in the level 4 Neonatal Intensive Care Unit (NICU) at Covenant Children's Hospital $(\mathrm{CCH})$. The outcome variables measured are compared to a national quality database (pedi NSQIP) to identify areas that may require programmatic development.

This study is a retrospective chart review performed at $\mathrm{CCH}$ for NICU patients undergoing general pediatric surgical procedures during their NICU stay from 4/1/17-12/31/19. Outcome variables assessed include length of stay, time to full feeds, ventilator days, complications, and unplanned return to OR. Demographic data such as gestational age at birth, birthweight, and associated anomalies have also been collected.

\section{Rodan Devega}

\section{Potential Health Effects of Bioactive Compounds on Depression: A Review of the Literature}

Rodan Devega, Daniel Payberah, Chanaka Kahathuduwa, Chwan-Li Shen

Major depressive disorder (MDD) is a mood disorder that is common in societies and cultures around the world. In the U.S. alone, an estimated 17.3 million adults have had at least one major depressive episoderepresenting $7.1 \%$ of all U.S. adults. The prevalence of major depressive episodes is higher among adult females $(8.7 \%)$ compared to males $(5.3 \%)$. Risk factors associated with MDD include trauma, medications, family history of depression, etc. It is characterized by feelings of persistent sadness, anhedonia, fatigue, suicidal ideation, etc. Current treatments for depression aim to alleviate symptoms via pharmacotherapy, psychotherapy, or a combination of the two. However, many medications involved with treating depression are accompanied by serious side-effects that have yet to be eliminated, such as sexual dysfunction, sedation, and weight gain. Because of these side-effects, studies have been performed to uncover the potential anti-depressant effects of less harmful bioactive compounds, so that they may be used in the treatment of MDD. Ultimately, this review describes the effects of common bioactive compounds, such as curcumin, saffron, garlic, resveratrol, tea, and omega- 3 fatty acids on depression and highlights their potential mechanisms of action on the molecular level; which involves a combination of up-regulating antioxidant (ex. catalase and glutathione enzymes) and anti-inflammatory (ex. IL-10) pathways and down-regulating pro-inflammatory (ex. TNF-alpha, COX-2) pathways. For example, garlic was shown to inhibit MAO inhibitors and stimulate catecholamine synthesis; while curcumin was shown to inhibit pro-inflammatory pathways; and theaflavin was shown to stimulate antioxidant pathways. Overall, these results attenuated the symptoms of MDD, which were measured via experimental parameters such as free swim test (FST), tail suspension test (TST), etc. in animal models.

\section{Travis Dowdle}

\section{Initial CO2 Laser Treatment Practices for Hypertrophic Burn Scars Among Surgeons at ABA Burn Centers}

Travis Dowdle, Kaylee Schrader, Chris Bruce, Joshua Frost, John Griswold

Background: Scarring is a major clinical outcome of severe burn wound healing. Severe scars often persist and significantly diminish quality of life by disfigurement, pain, itchiness, and contractures restraining body and joint movement. In the last decade, laser therapy has become a popular treatment modality for severe burn scars, particularly the use of ablative fractional carbon dioxide (CO2) lasers.

Introduction: The efficacy of $\mathrm{CO} 2$ lasers for the treatment of hypertrophic burn scars has been established via systematic review and international guidelines. There is currently intense multidisciplinary interest regarding laser treatment utilizing $\mathrm{CO} 2$ laser therapy. However, there have been no attempts to query the $63 \mathrm{ABA}$ (American Burn Association) centers across the United States regarding specific treatment parameters involving serious, sometimes high, total body surface area (TBSA) burns.

Methods: A Qualtrics survey consisting of 14 questions was administered to burn surgeons practicing at all 63 ABA burn centers across the U.S. Topics 
were assessed such as specific laser parameters (5), treatment preferences (2), peri-operative follow-up (5), scar assessment practices (1), and TBSA treatment tolerance (1).

Results: Exploratory, descriptive data was analyzed in collaboration with the Texas Tech University Health Sciences Center Biostatistics Department. Surgeons practicing at 27 of the 63 total ABA burn centers responded to our survey (43\% response rate). Data elucidates the level of variance regarding current initial management of hypertrophic burn scars via $\mathrm{CO} 2$ laser treatment.

Conclusion: Our findings allow surgeons to study how their $\mathrm{CO} 2$ laser practices for hypertrophic burn scars compare to those of their colleagues at other ABA affiliated burn centers. Standardization of care when utilizing ablative fractional $\mathrm{CO} 2$ lasers should be further explored.

\section{Abigail Ellington}

\section{Documenting and Describing Irritable Bowel Syndrome on Reddit}

Abigail Ellington, Sameer Islam

The purpose of this study is to determine what people are posting on the internet page Reddit regarding their struggles with Irritable Bowel Syndrome. This will allow healthcare providers to have a better sense of what major concerns patients have and how to better address their needs. Using Reddit Pushshift API, posts on the subreddit page for IBS from May 2020 were accessed using seven different filters. These posts were then categorized by the tags specified in the post and subjectively by content. It was found that most of the posts were looking to discuss and ask questions regarding symptoms and symptom management. Additionally, many users did not include a tag in the posts; however, those that did most often used the tag "question." Major conclusions that can be drawn from this study are that patients do not feel that their health care provider is taking their concerns seriously and that patients are not fully aware that IBS is different from patient to patient.

\section{Adrian Falco}

\section{Effectiveness of Student-Run Free Clinic Elective for Medical Students}

\author{
Adrian Falco, Brianna Marschke, Fiona Prabhu
}

Student-run clinics (SRCs) generally serve homeless, at-risk, and low-income populations for free or little cost. In addition to the benefits of providing care for vulnerable populations, SRCs provide students the ability for professional growth and increased patient and interprofessional communication skills. Many SRCs provide an elective for medical students that want to learn, volunteer, and care for at-risk populations; however, the effectiveness of these electives has scarcely been studied. First and second year medical students at the Texas Tech Health Sciences Center School of Medicine volunteered to participate in the Free Clinic Elective $(n=19)$ which included five lectures and took place during the Fall 2020 semester. Although the sample size is limited, students reported the elective was an effective use of time and improved verbal and written communication skills as a medical student working in the SRC. Students also reported feeling more comfortable treating underserved populations such as the homeless, LGBTQ, POC and better able to define and identify how social determinants can impact the health of patients. Questions assessing the students' knowledge over the content of the material presented (paired-t test) generally presented with no significant difference between pre- and post-quizzes. This lack of difference could possibly be attributed to the low difficulty level of the questions.

\section{Bernardo Galvan}

\section{Classic Clinical Descriptions of Disease: Curing Medical Education with a Dose of the Past}

Bernardo Galvan, Katherine Holder, Laxmi Chintakayala, Sonia Khan, Erin Choi, Steven Berk

The importance of clinical skills, including obtaining patient history and performing physical examination, has been de-emphasized in the modern medical school curriculum. With advancements in diagnostic technologies, the clinical presentation of diseases in 
medical textbooks has been simplified, diminished, and largely replaced with detailed pathophysiology and laboratory findings. The implementation of the United States Medical Licensing Exam (USMLE) Step 1 has also contributed in pushing medical education towards classroom-based learning rather than clinical experience. Clinical skills competency is crucial to accurately diagnose patients and simultaneously lowers health care costs by not relying on unneeded diagnostic tests. To address this gap in medical knowledge, a group of students at Texas Tech University Health Sciences Center have assembled a website documenting classic clinical disease descriptions written by some of the renowned physicians from the 19th and 20th centuries including Osler, Flint, Gowers, and many more. This website will continue to grow, and will hopefully be a useful tool to professors, physicians, and medical students around the nation.

\section{Ana Garcia}

\section{Chronic Depression and Prescription Likelihood as a Function of Race and Sex in a Low Income Clinic Sample}

Ana Garcia, Billy Ulyses Philips Jr, Catherine Hudson, Michael Penuliar

Background: The TTUHSC Free Clinic provides free healthcare to the uninsured population in Lubbock and surrounding area. This study provides insight into the frequency and reason for patient visits to the TTUHSC Free Clinic from June 2019-July 2020. This information was used to delve into the relationship between demographic factors of uninsured patients in West Texas and their health.

Procedure: Data came from the medical charts of patients that visited the TTUHSC Free Clinic from June 2019-July 2020. The information recorded included patients' total visits, race/ethnicity, income level, chronic conditions, chief complaints, and prescribed medications. Two logistic regression models of 460 patients were performed and analyzed.

Results: One model showed that race and gender contributed to a higher likelihood of chronic depression history. Analysis of the Free Clinic's patient data indicated that White patients were more likely to report a history of chronic depression than Hispanic and Black patients. Females over males were also more likely to report a history of chronic depression. A second model looked at the likelihood of prescribing antidepressants to patients with chronic depression. This found that Hispanics without a history of chronic depression were less likely than White patients with a similar mental health history to receive anti-depressants during their visit. There were no significant differences between White and Black patients.

Conclusion: The results showed that demographic of ethnicity and sex within a low-income population may be uniquely tied to chronic depression and the prescription of antidepressants. Increased research on free clinics can provide insight into the obstacles facing their patients. For example, Hispanic patients may not be getting the medicines they need due to stereotyped thinking or other factors. This information may lead to a better understanding of the role demographic factors play in determining diagnosis and treatment.

\section{Holly Grossman}

\section{Influence of Environmental Contaminants on Patients Undergoing IVF}

LL Penrose, S Khalili, D Leung, K Ahmad, SD Prien, H Grossman, E Fine

Objective: In past studies covering the impact of environment on fertility, differences were demonstrated in the number of available sperm, oocytes recovered, healthy oocytes, and atretic oocytes in patients living in rural and urban environments. However, fertilization rates and pregnancy outcomes were not influenced, suggesting that environmental factors may influence oocyte quality. As pollutants can affect hormone cascades, the objective of this study is to determine if hormone profiles differ in patients undergoing assisted reproductive technologies (ART) procedures in urban and rural environments.

Methods: A chart review evaluating hormone profiles for ART procedure reports from 2014-2017 (N = 163) was categorized into urban, mainly urban, mainly 
rural, and rural populations based on zipcode. Data included precycle profiles, down-regulation, maximum estrogen levels, and pregnancy outcomes. Data were recategorized between different environmental regions based on agrochemical use, grouped to correspond to periods of heavy agrochemical use, and analyzed for each hormone. Data were compared by ANOVA, independent t-test, or Chi-square.

Results: No differences were found between the patient hormonal profiles for any hormone reviewed, regardless of their residential environment $(p=0.118)$. Additionally, no difference was found between the hormonal profile of patients from different agricultural regions where agrochemical used varied in intensity $(p=0.077)$. Finally, no differences were seen in the hormonal profiles of patients undergoing treatment cycles during periods of intense versus minimal agrochemical use $(p=0.127)$.

Conclusion: Current data suggest that changes in oocyte quality are independent of hormonal profile and may be influenced during the hormone-independent stage of follicular development as follicles progress from the primordial to the primary stage of development.

\section{Delton Hall}

\section{Implementing a Fast-Track Extubation Protocol For Patients That Have Undergone Cardiac Surgery}

Delton Hall, Cooper W Phillips, Jinesh

Lachmansingh, Tyson Verhaal

Fast-track extubation is the process of extubating patients that have undergone surgery within six hours post-operative. Fast-track extubation involving cardiac surgery is a relatively new concept, with one of the first studies emerging in 2013. Multiple studies have aimed for increasing hospital efficiency by decreasing mortality, hospital length of stay, adverse events, etc. However, the data is conflicting with previous research articles showing improvements in the variables listed previously and others showing no significant difference. The goal in this research study was to first construct an anesthesia protocol that was safe for patients and that reduced time to extubation to under six hours post-operative. Patients were selected for fast track extubation prior to the surgery; furthermore, the course of the operation ultimately determined if a patient was a candidate for fast track extubation. This approach reduced selection bias and prioritized patient safety. The control group received the same anesthetic approach, while the intervention group received an anesthetic approach that was tailored to the individual patient. Methadone was given before surgery by mouth in the intervention group if patients were able to tolerate the medication. Once in the ICU, the intervention group received Dexmedetomidine instead of Midazolam. An extubation deadline card was placed on ventilators as a reminder for time to extubation. Extubation was cleared by the ICU physician on staff, using general weaning parameters, and extubation was primarily done by the respiratory therapist on staff. Data shows that the control group $(n=73)$ had an average time to extubation of 481 minutes (8h $1 \mathrm{~min})$. The intervention group $(\mathrm{n}=27)$ had an average time to extubation of 347 minutes ( $5 \mathrm{~h}$ $47 \mathrm{~min}$ ). Using a t-test analysis assuming unequal variances, the data has a p-value of .002 . There is significant difference in time to extubation when the interventions mentioned are applied.

\section{Neil Jain}

\section{Biomolecular Endotype Factors Involved In COVID-19 Airway Infectivity}

Neil Jain, Rahul Varman, James Tarbox, Tam Nguyen

Objectives: To review the current knowledge of biomolecular factors surrounding otorhinolaryngeal illnesses and analyze their presence in COVID-19 virulence. Emphasis was placed on cytokines and vitamin $D$ for determining susceptibility of illness.

Methods: A primary literature search of PubMed and Google Scholar for articles published between January 1, 2002 to May 31, 2020, was performed without language restrictions from May 8, 2020 to May 31, 2020. A focused second search was conducted from October 31, 2020 to November 2, 2020 for articles published between January 1, 2002 to October 31, 
2020. Eligible articles were selected after evaluation of titles, abstracts, and references. A total of 45 were included in this review.

Results: Differing endotype classification schemes are used to determine cytokines present in chronic rhinosinusitis, asthma, and allergies. While immunologic responses and biomarkers are primary methods of differentiation, recent literature has also implicated geographic distribution of chronic rhinosinusitis patients in accounting for cytokine variations. The cytokines of interest (IL-4, IL-13, and INF- $\gamma$ ) present in the endotypes of these conditions may point towards protective mechanisms against COVID-19 through downregulation of the ACE2 receptor. These cytokines and Vitamin D highlight new areas of study for factors affecting SARS- CoV-2 virulence.

Conclusions: Further research is needed to understand the effects of Vitamin $D$ and the various cytokines prevalent among endotypes of nasal/ pharyngeal illnesses on COVID-19 pathogenesis. Findings may point towards epidemiologic trends of SARS-CoV-2 transmission and have future therapeutic indications.

\section{Abdurrahman Kharbat}

\section{Bilateral Asymmetry in the Upper Extremity}

\section{Abdurrahman Kharbat, Cameron Cox, Brendan} Mackay

In living patients, traumatic injury or tumor resection can create length alterations in the upper extremity (UE) bones requiring reconstruction. It is critical to maintain anatomical length of an injured limb to restore normal function. Direct measurement of the contralateral bone is often used for target length of reconstruction, but does not account for potential asymmetry. Given the importance of accurate length estimations and the paucity of data on living populations, we created a study to evaluate asymmetry of UE long bones by analyzing radiographic images of living patients. Bilateral X-ray images previously taken for either trauma or chronic osseous conditions were collected for subjects ages 2-81. After screening, 61 patients were included (19 humerus, 28 radius, 29 ulna). Three length measurements were taken of each bone. Median length of 3 serial measurements was used for analysis. Bootstrapping was performed to reach sample sizes of 200,500, and 1000. Wilcoxon signed-ranks tests were performed to determine whether contralateral bones differed significantly in length. Mean absolute differences: $27.0 \mathrm{~mm}$ humerus, $8.6 \mathrm{~mm}$ radius, $7.5 \mathrm{~mm}$ ulna. There was no clear trend favoring a longer side in any bone. $57.9 \%$ $(11 / 19)$ had a right humerus longer than left, $60.7 \%$ (17/28) longer right radius, and 48.3\% (14/29) longer right ulna. Wilcoxon Signed Rank tests: No significant differences between contralateral bones in direct measurement groups. In bootstrap samples, significant length differences were found in all sample sizes for the humerus and radius, but only in the 1000 sample for the ulna. Although the exact threshold is unclear, length alteration may negatively impact UE motor function and/or induce pain. In current reconstructive algorithms, asymmetry is not well accounted for. The data we present may help to develop improved length estimation algorithms, ultimately leading to improved patient outcomes.

\section{Elleana Majdinasab}

\section{Polymer-Encased Nanodiscs and Polymer Nanodiscs: New Platforms for Membrane Protein Research and Applications}

Angela Chen, Elleana Majdinasab, Mariana Fiori, Hongjun Liang, Guillermo Altenberg

Membrane proteins (MPs) are essential to many organisms' major functions. They are notorious for being difficult to isolate and study, and mimicking native conditions for studies in vitro has proved to be a challenge. Lipid nanodiscs are among the most promising platforms for MP reconstitution, but they contain a relatively labile lipid bilayer and their use requires previous protein solubilization in detergent. These limitations have led to the testing of copolymers in new types of nanodisc platforms. Polymer-encased nanodiscs and polymer nanodiscs support functional MPs and address some of the limitations present in other MP reconstitution platforms. In this review, we provide a summary of recent developments in the use of polymers in nanodiscs. 


\section{Brianna Marschke}

\section{Review of Noninvasive Imaging to Assess Chemotherapy-Induced Neuromuscular Disorders}

Brianna Marschke, Suhas Pol, Kishor Bhende

Background: Chemotherapy-induced peripheral neuropathy (CIPN) is a type of neuromuscular disorder that commonly negatively affects the quality of life of cancer patients. There are many different imaging techniques used in the diagnosis and assessment of CIPN, including EMG, muscle biopsy, MRI, and ultrasound. Although it is clinically important to monitor the extent of peripheral nerve damage to better treat patients, it is difficult to assess children due to their limited speech development and the invasive modality of some of the invasive imaging techniques. The purpose of this article is to review and report current knowledge of imaging techniques used to assess CIPN.

Methods: We conducted a retrospective review study at an academic medical center to identify specific features of chemotherapy- induced neuromuscular disorders. We performed a literature search via 'Pubmed' for relevant literature published in English from 2000-2020 using keywords "chemotherapyinduced peripheral neuropathy," "neuromuscular disease," and "diagnostic imaging." We included human studies of clinical trials and randomized controlled trials. In total, we analyzed 111 articles.

Results: Whereas imaging to assess, monitor, and diagnose CIPN in adults has been more thoroughly researched, imaging techniques that are successful and effective in children are scarce. Although progress has been made in identifying that the current gold standard of EMG and NCS to diagnose and monitor CIPN in pediatric populations is insufficient, there is still not an adequate technology that exists.

Conclusion: There is need for the expansion of noninvasive imaging techniques that are well-suited for children. New noninvasive imaging techniques that appear promising are still in early stages of development and will require significant advancements and trials to ensure their effectiveness, accuracy, sensitivity, and specificity.

\section{Kirby Mateja}

\section{Pediatric Trauma Management: Development of Whole Blood Transfusion Protocols}

Kirby Mateja

Background: Historically, whole blood transfusions have been an effective treatment for patients suffering massive hemorrhage following trauma. Despite whole blood successes, it presents major logistical problems for hospitals in terms of collection and storage and has been shown to increase the risk of transfusion-transmitted disease and plasma-associated transfusion reactions. The 1970s saw a rise in component therapy with a targeted ratio of packed red blood cells, platelets, and plasma which became the norm in transfusion protocols across the world despite the drawbacks of using a more dilute blood mixture. Recently, there has been a resurgence in the use of whole blood replacement therapy in areas of the world with reduced access to component therapy. Preliminary studies have indicated that whole blood, despite the aforementioned drawbacks, may actually be the optimal resuscitation fluid for massive hemorrhage. Whilst several studies examine the use and establishment of whole blood transfusion protocols in adults, especially in military members, there is a paucity of information surrounding the use in children. Trauma and resultant hemorrhage are some of the leading causes of medically preventable death in children over the age of one. This retrospective study examines the use of replacement therapy in pediatric trauma cases in order to develop protocols and determine the resources needed.

Methods: This study examined the reason for administration, the amount of blood used and the outcomes of all pediatric trauma patients who either received a blood transfusion, required activation of massive transfusion protocol, or had emergency release blood available at Covenant Children's Hospital from 4/1/17-12/31/19. This information will be used to establish protocols outlining the use of whole blood transfusions in children.

Results: at the time of abstract submission the data was being analyzed by the CRI. 


\section{Adin Mizer}

\section{Orthopaedic Surgery Total Joint Registry- Preliminary Outcomes of Knee and Hip Scores}

Adin Mizer, Albin John, Stephen Rossettie, Bernardo Gonzalez, Elizabeth Eichman, Amanda Purcell, George Brindley

Introduction: The focus of this Total Joint Registry (TJR) is to provide more information about the best methods of treatment to improve outcomes total hip, knee, and shoulder replacement recipients. Over time, this registry will provide information about the summation of one's physiology, comorbidities and implant technology on surgical outcomes.

Methods: The population consists of patients of TTUHSC Orthopaedic Surgery receiving a primary total hip, knee, or shoulder arthroplasty by Dr. George Brindley, MD; Dr. Mark Jenkins, MD; Dr. Cyrus Caroom, MD; or Dr. Mathew Ferguson, MD. Data is collected pre-operation (pre-op), 6 weeks, 1 year, 3 years, 5 years and 10 years post-operation (post-op). A SF-36 health questionnaire is completed at each collection point and a Knee Society Score, Harris Hip Score or DASH Shoulder score is determined for patients receiving a knee, hip or shoulder replacement, respectively. Additionally, at the time of entry into the registry, the patient's comorbidities and pre-operative status are entered into the spreadsheet.

Results: For patients undergoing a total hip arthroplasty there was a statistically significant positive relationship between the change in pre-op to post-op hip scores and those without cardiac comorbidities. Despite difficulties presented by COVID-19, a sustainable operation and workflow for collection of patient information on this registry has been established. The presented preliminary data shows the potential of this registry in providing vital information to better understand predictive factors in total joint replacement surgery. The goal is to continue to expand the registry and contribute to improving patient outcomes.

\section{Erin Morris}

\section{Global Patterns and Outcome of Necrotizing Soft Tissue Infections: A Systematic Review and Meta-Analysis}

Samudani Dhanasekara, Brianna Marschke, Erin Morris, Chanaka Kahathuduwa, Sharmila Dissanaike

Objective: We performed the first systematic review/meta-analysis on published reports of necrotizing soft tissue infections (NSTIs) from across the globe.

Summary of Background Data: Frequency, microbiology, and outcomes of NSTIs vary based on locoregional and environmental factors; however, there has been no global survey of these patterns.

Methods: Peer-reviewed empirical studies examining rates of polymicrobial and monomicrobial NSTIs with microbial isolation were extracted along with geographical location using PubMed, Scopus, ProQuest, and Web of Science. Random-effects metaanalyses were performed and adjusted for publication bias. Meta-regression analyses examined moderator effects of risk factors.

Results: One-hundred and five studies (8718 total patients) were included. Pooled prevalence of polymicrobial and monomicrobial infections were $53 \%$ and $38 \%$, respectively. Truncal NSTIs were commonly polymicrobial $(p<0.001)$, while monomicrobial infections prevailed in extremities $(p=0.008)$. Global prevalence of monomicrobial NSTIs was observed to increase by $1.1 \%$ annually $(p=0.003$ ). Staphylococcus aureus emerged as the commonest organism globally, as well as in North America, Asia, Middle East and Africa, followed by Streptococcus pyogenes and Escherichia coli. Methicillin-resistant Staph. aureus (MRSA) accounted for $7.5 \%$ of NSTIs globally. Overall mortality rate was $18 \%$, with no difference based on geographic region. Mortality has been decreasing globally over the last two decades $(p=0.052)$.

Conclusions: Though polymicrobial infections remain predominant worldwide, the incidence of monomicrobial infections is rising. MRSA infections are less common than previously reported. Overall mortality is lower than many prior reports. No mortality differences were noted between regions, despite major variations in available healthcare resources. 


\section{Cristina Natha}

\section{Connexin Hemichannel Inhibitors Based on Aminoglycosides}

Cristina Natha, Varun Vemulapalli, Mariana Fiori, Cheng-Wei Chang, Guillermo Altenberg

Connexins are membrane proteins involved directly in cell-to-cell communication through the formation of gap-junctional channels. These channels result from the head-to-head docking of two hemichannels, one from each of two adjacent cells. Undocked hemichannels are also present at the plasma membrane where they mediate the efflux of molecules that participate in autocrine and paracrine signaling, but abnormal increase in hemichannel opening can lead to cell damage in disorders such as cardiac infarcts, strokes, deafness, cataracts, and skin diseases. For this reason, connexin hemichannels have emerged as a valid therapeutic target. Traditional hemichannel inhibitors are not ideal leads for the development of better drugs for clinical use because they are not specific and/or have toxic effects. Newer inhibitors are more selective and include connexin mimetic peptides, anti-connexin antibodies and drugs that reduce connexin expression such as antisense oligonucleotides. Re-purposed drugs and their derivatives are also promising because of the significant experience with their clinical use. Among these, aminoglycoside antibiotics developed in our laboratory have been identified as inhibitors of connexin hemichannels that do not inhibit gap-junctional channels. Here, we discuss connexin inhibitors with a focus on aminoglycoside antibiotics and our derivatives of kanamycin A that inhibit connexin hemichannels, but do not have antibiotic effect.

\section{Tommy Ngo}

\section{Outcomes of Peripheral Nerve Repair in Non- transected and Partially Transected Nerves: A Review of the Literature}

Cody Perry, Cameron Cox, Tommy Ngo, Alexis Rounds, Brendan MacKay

Introduction: Peripheral nerve injuries (PNIs) are common and present with varying symptoms depending on the severity and nerves involved. While the mechanisms of nerve injury and regeneration have been well-described, current treatment algorithms fail to consistently achieve full functional recovery. Ineffective treatments for PNIs can result in disabling and long-lasting, or even irreversible complications for patients. The most prominent direct complications include chronic pain, hyperesthesia, cold intolerance, and compromised function secondary to motor and/ or sensory deficits in the affected extremity. When patients are unable to return to full functional capacity, downstream effects such as loss of employment and cost of continued medical care may amplify reductions in quality of life and adverse psychological outcomes.

Methods: We conducted a systematic review of Pubmed and Google Scholar in accordance with PRISMA guidelines to define the current understanding of partial nerve injury presentation, non-surgical treatment, surgical repair techniques, and patient outcomes. Search words included "peripheral nerve injury", "nerve repair", "non-transected", "partial-transection", or "lesion incontinuity", or "neuroma-in-continuity".

Results: Several studies have been published that show moderate success for upper extremity in-continuity lesion repaired surgically, as a small majority of patients achieved at least a moderate (LSUHSC Grade of 3) degree of recovery. In addition, multiple reports demonstrated better outcomes for patients with intact nerves that were treated by neurolysis; or in some cases even complete transection repairs had better results.

Conclusion: There is no consensus gold standard on optimal approach to non-transected nerve injuries and treatment algorithms continue to evolve. Given the paucity of clinical data, this review may serve as a resource for physicians treating these difficult injuries.

\section{Shree Patel}

\section{Lidocaine and Ketamine Use to Treat Procedural Pain in Burn Patients}

Shree Patel, John Wall, Travis Dowdle, Vanessa Ku, Josh Frost, Ebrahim Payberah, John Griswold 
Introduction: Burn patients undergo repeated wound care procedures during their hospital stay. This can intensify the pain of their burn injury, which may develop into chronic pain. The proper management of procedural pain in burn patients improves quality of care and reduces procedure associated anxiety and distress. In this study, we will explore the use of analgesics such as lidocaine and ketamine as an alternative method to treat procedural pain compared to the traditional use of opioids.

Methods: Patients admitted to the UMC burn ward were administered IV ketamine and lidocaine or an opioid treatment prior to wound care treatments. Patient demographics, total burn surface area, verbal and non-verbal pain, and vitals were documented before, during, and after wound care treatments. Any lidocaine and ketamine associated complications were documented in patients post-treatment.

Results: Patients receiving the lidocaine and ketamine drug combination only reported significantly elevated pain scores at five minutes into the wound care procedures compared to patients receiving opioids. There was no significant difference between patients receiving the lidocaine and ketamine combination and patients receiving opioids in displayed levels of non-verbal pain behaviors. In the fifteen minutes prior to the wound care procedure through the first five minutes of the procedure, patients receiving the lidocaine and ketamine combination maintained increased heart rates compared to control morphine patients.

Discussion: Results from the study suggest that the combination drug therapy of lidocaine and ketamine had similar analgesic effects to traditionally used opioid therapeutics in the management of burn patient pain during wound care procedures. This indicates that the lidocaine and ketamine combined therapy may be a useful alternative to traditional opioid therapy use in reducing procedural pain in burn patients, thereby potentially lowering opioid dependency.

\section{Daniel Payberah}

\section{The Effectiveness of Botulinum Toxin in the Prevention and Treatment of Tension-Type}

\section{Headaches: A Systematic Review and Meta-Analysis}

Daniel Payberah, Joanna Chyu, Chathurika

Dhanasekara, Chwan-Li Shen, Chanaka Kahatuduwa

Background: Tension-type headache (TTH) is the most common type of headache, with a lifetime prevalence up to $78 \%$. Management of chronic TTH that is resistant to analgesics remains a challenge. Botulinum toxin type A (BTX-A), a paralytic used in treating migraines, remains an uncertain treatment for TTH. This study aimed to investigate the efficacy of BTX-A as a treatment on isolated TTH in a systematic review and meta-analysis.

Methods: PRISMA guidelines were followed. PubMed, Scopus, Web of Science, and ProQuest databases were searched to collect records regarding BTX-A and TTH, without language or date restrictions. Two independent reviewers used predetermined eligibility criteria to systematically screen records to include randomized, controlled clinical trials testing the efficacy of BTX-A on treating TTH. Eligible articles were assessed for risk of bias per the Cochrane handbook. Pre- vs. post-intervention intensity and frequency of TTH and the proportion of responders to treatment were extracted from intervention and control groups. DerSimonian Liard random-effects meta-analyses were performed using the meta package in $R$ (4.0.2).

Results: Eight trials were included, totaling 564 study participants. Overall risks of bias were mixed, with most studies being low risk. Compared to placebo, BTX-A treatments showed improvement in headache intensity (Cohen's $d=-0.81[-1.56,-0.07]$, $\mathrm{n}=262$ participants, $\mathrm{I}^{2}=86 \%$ ), a decrease in headache frequency $(\Delta=-2.92$ days/month $[-4.43,-1.41]$, $\mathrm{n}=205$ participants, $\mathrm{I}^{2}=51 \%$ ), and a $64 \%$ greater probability of observing improvement in TTH (RR = $1.638[1.075,2.495], \mathrm{n}=305$ events, $\mathrm{I}^{2}=15 \%$ ).

Conclusion: While the results on the efficacy of BTX-A on TTH are promising, our results are limited by the high between-study heterogeneity, limited sample sizes, and the limited number of high-quality controlled trials. Future well-designed, adequately- powered, multicenter randomized controlled trials are warranted. 


\section{Mohammed Pourghaed}

\section{Association of Vitamin D insufficiency/deficiency with depression, obesity, and diabetes in an ethnically diverse population in West Rural Texas-A follow-up analysis of Project FRONTIER}

Mohammed Pourghaed, Felipe Ramirez Velandia, Ashish Sarangi, John Culberson, J. Josh Lawrence

Vitamin D (VD) deficiency (VDD; $\leq 20 \mathrm{ng} / \mathrm{mL}$ ) and insufficiency (VDI; 21-29 ng/mL) are associated with numerous health disparities. Dysfunction in two major pathways lead to VDD/VDI: 1) failure to consume, absorb, and/or transport VD and 2) failure to produce VD via physiological processes. Expanding on a study by Johnson et al. (2010), we tested the hypothesis that a significant association exists between VD level and depression, Hispanic ethnicity, and comorbidities. Using 299 participants from Project FRONTIER, we examined the relationship between VD and depression using the using the Geriatric Depression Scale (GDS) and its four subfactors: apathy, cognitive impairment, dysphoria, and meaninglessness. Statistical comparisons were performed us ing Prism 8/9. We found that 184/299 (61.5\%) had VDD/VDI. VD level correlated with GDS Total score $(r=-0.1333, p=0.021)$; dysphoria $(r=-0.19$, $p=0.001)$ and meaninglessness subfactors $(r=-0.15$; $p=0.011)$ contributed too. Demographic analysis using logistic regression revealed that Hispanics $(n=114)$ had lower VD level $(p<0.0001)$ and higher depression $(p<0.0001)$ than non-Hispanics $(n=178)$. A correlation matrix revealed relationships between VD level and three co-morbidities: obesity $(p=0.020)$, diabetes $(p=0.002)$, and nicotine use $(p=0.00022)$, as well as VD $(p<0.0001)$ and multivitamin $(p<0.0013)$ supplementation. Finally, using these variables, a multivariate regression model predicted VD level $(R 2=0.62)$. VD levels were associated with depression; GDS dysphoria and meaninglessness subdomains were the highest association. Collectively, our findings identify Hispanics at risk for VDD/VDI, especially those with co-morbidities. Moreover, high sufficient VD levels $(95.5 \mathrm{ng} / \mathrm{ml})$ predict optimal reduction of depression symptoms in this aging population. While the causal underlying mechanisms remain to be examined, this study provides strong rationale for screening for VDD/VDI when evaluating depressive symptoms in this aging population.

\section{Stefan Raicevic}

\section{Creating a Cardiogenic Shock Database for West Texas and Eastern New Mexico: Plan for Retrospective Study and Analyze Lone Star Registry}

Stefan Raicevic, Christopher Le, En Dien (Sam) Lao

Cardiogenic shock (CS) is a high acuity and hemodynamically diverse state of end organ hypoperfusion that is associated with multisystem organ failure. CS is pragmatically defined as a state in which ineffective cardiac output is caused by a primary cardiac disorder such as impairment in myocardium, valvulopathy, conduction disease, or pericardial disease. It is a clinical diagnosis characterized by persistent hypotension unresponsive to volume replacement accompanied by end organ hypoperfusion requiring intervention with pharmacological and/or mechanical support. Despite improving survival in recent years, patient morbidity and mortality remains high.

We aim to describe the clinical and procedural characteristics and outcomes of patients with cardiogenic shock who underwent mechanical hemodynamic support at the TTUHSC-University Medical Center from January 2015 to present. We will also compare certain clinical patient and procedural characteristics between patients with cardiogenic shock who underwent treatment with medical management without mechanical support.

Our methods involve retrospective chart review of all patients with cardiogenic shock treated with and without mechanical support. Mechanical support options analyzed include: intra-aortic balloon pumps, Impella 2.5, CP, RP and 5.0, and extracorporeal membrane Oxygenation (ECMO). We have built a database with clinical and procedural characteristics and plan to apply descriptive statistical analysis as well as ANOYA and logistic regression to compare between groups. The study will add to the body of knowledge regarding the utilization rate, feasibility, efficacy, and safety of temporary mechanical support in patients 
presenting with cardiogenic shock. This will be the first database study of its kind in West Texas and Eastern New Mexico. This will allow us to better understand the dynamics of cardiogenic shock in our community, so we can apply better treatment protocols.

\section{Malvika Ramesh}

\section{Rlip loss poses a therapeutic target for treatment of Atopic Dermatitis}

M Ramesh, JA Tarbox, S Singh, S Awasthi

Rationale: Rlip knockdown protects p53 deficient mice from carcinogenesis and reduces inflammation. In Rlip null mice, increased oxidative stress alone was not enough to increase inflammation. Thus, Rlip is necessary to translate oxidative stress into inflammation. Rlip knockdown disrupts inflammatory signaling in atopic dermatitis through altering Th1/Th2 immune genes. Here we review potentially significant genes by analysis of RNA sequence pathways in a previously unknown role, atopic dermatitis, in relation to partial Rlip loss.

Methods: RNA sequencing (RNA-Seq) was conducted in wild-type and Rlip loss mice liver. RNA sequencing runs were performed in a Illumina HiSeq 2500 platform with HiSeq SBS V4 kits, and reads were aligned using Tophat v2.0 to mouse reference genome $\mathrm{mm} 9$.

RNA-Seq and genome analysis lead to the identification of various canonical pathways upregulated/ downregulated by Rlip loss. Z scores, expressing the magnitude of regulation in a positive/negative manner, were attributed to each gene. Analysis of existing literature was conducted to identify new roles for these Rlip loss affected genes.

Results: Analysis of RNA-Seq shows the top differentially expressed upregulated genes involved in immunology canonical pathways are IL-13RA2, ACVR1C and ALDH3A1 with Z scores of 2.127, 2.107 and 1.947 respectively.

Conclusions: RNA-Seq analysis shows genes involved in immunologic pathways and inflammation are among the top affected by pharmacologic knockdown of Rlip. Upregulation of canonical pathways including IL-13RA2, ALDH3A1 and ACVR1C through
Rlip inhibition could serve a therapeutic target for the treatment of atopic dermatitis through reduction of inflammation. Further studies are necessary to fully understand the mechanism behind this process.

\section{Nikita Rao}

A literature review of repetitive transcranial magnetic stimulation for the treatment of depression during pregnancy

\author{
Nikita Rao, Michelle Terry, Manish Kumar, Regina \\ Baronia
}

Background: Patients with prepartum depression are at increased risk for experiencing negative obstetric and neonatal outcomes. Repetitive transcranial magnetic stimulation (rTMS) is a safe and effective non-invasive treatment for major depressive disorder. However, data supporting safety and efficacy of use during pregnancy is limited. The purpose of this literature review was to synthesize current findings on rTMS as a mode of treatment for major depression during pregnancy.

Methods: PubMed, SCOPUS, and Web of Science databases were searched for the following keywords: transcranial magnetic stimulation, major depressive disorder, pregnancy. 32 peer-reviewed papers were screened for meeting inclusion/exclusion criteria; 13 were selected for analysis.

Results/Conclusions: rTMS appears to be a relatively safe and effective option for treating and maintaining remission of depressive symptoms during pregnancy. Current findings are limited, but show minimal adverse birth outcomes and treatmentassociated side effects. More large-scale clinical studies are needed to further elucidate the benefits and drawbacks of rTMS as a treatment option for major depression during pregnancy.

\section{Sanjana Rao}
Analysis of Glucose Levels in Patients Hospitalized With COVID-19 During the First Phase of this Pandemic in West Texas


Sanjana Rao, Kiran Ali, Jeff Dennis, Gilbert Berdine, Victor Test, Kenneth Nugent

Background: Patients with hyperglycemia during hospitalization, especially during ICU hospitalizations, often have worse outcomes, even if they do not have a premorbid diagnosis of diabetes. High glucose levels can provide insight into the underlying pathogenesis of a disease and can contribute to tissue injury. Some patients with COVID-19 have hyperglycemia during hospitalizations.

Methods: The Infectious Disease and Control office at University Medical Center in Lubbock, Texas, provided a list of patients with a COVID-19 infection hospitalized between March 1 and May 15, 2020. The medical records were reviewed to collect information on age, gender, history of diabetes, and glucose levels on admission and through the first 7 days of hospitalization.

Results: This study included 63 patients with a mean age of $62.1 \pm 14.1$ years. Thirty-five patients $(55.6 \%)$ were males. The inhospital mortality rate was $30.2 \%$. The mean admission glucose level was $129.4 \pm 57.1 \mathrm{mg} / \mathrm{dL}$ in patients who survived $(\mathrm{N}=$ $47)$ and $189.6 \pm 112.2 \mathrm{mg} / \mathrm{dL}$ in patients who died during hospitalization $(\mathrm{N}=16, \mathrm{P}=.007)$. An admission glucose greater than $180 \mathrm{mg} / \mathrm{dL}$ predicted mortality in a model adjusted for a diabetes, age, and male gender. The mean differences between the maximum and minimum glucose levels calculated over the first 7 days of hospitalization were $112.93 \pm 115.4(\mathrm{~N}=47)$ in patients who survived and were $240.5 \pm 97.7(\mathrm{~N}=15)$ in patients who died during hospitalization $(P=.0003)$. A difference between the maximum and minimum glucose level greater than $105 \mathrm{mg} / \mathrm{dL}$ was associated with increased mortality.

Conclusions: Patients who died during hospitalization for COVID-19 had higher admission glucose levels than patients who survived. Larger differences between maximum and minimum glucose levels during the first 7 days of hospitalization were associated with increased mortality. These results suggest that high glucose levels identify patients at increased risk for mortality and warrant more study.

\section{Madison Schoeberl}

\section{Vitamin D levels in pediatric patients undergoing chemotherapy}

Madison Schoeberl, Megan Mikkelson, Eduardo Urias, Chris Scott, Jaehoon Lee, Chwan-Li Shen, Mohamad M. Al-Rahawan

Vitamin $D$ is essential for bone and immune health. Children with cancer are at risk of vitamin D deficiency. This study investigates if children with cancer are vitamin $\mathrm{D}$ deficient compared to matched controls and whether vitamin D levels worsen throughout the course of their cancer treatment. This prospective cohort study enrolled 17 children with cancer, who at enrollment were expected to receive chemotherapy for 6 months, along with 16 age-and-ethnicity-matched controls without a diagnosis of cancer. The serum levels of 25-hydroxycholecalciferol vitamin D (25D) were assessed at baseline (T1, both cancer and control groups) and 3-4 months and 6-8 months later (T2 and T3, cancer group only). Bivariate tests and general linear modeling were utilized to compare 25D at baseline between the two groups. Multilevel modeling was conducted to examine the fluctuation of 25D over time in children with cancer accounting for their age, ethnicity, cancer type, and season of measurement. The participants were $9.2 \pm 4.8$ years old, with BMI of $20.1 \pm 6.3 \mathrm{~kg} / \mathrm{m}^{2}$. The majority were male $(61 \%)$ and Hispanic $(61 \%)$. The cancer and control groups did not differ in age, race, ethnicity, or BMI (all $p>0.05$ ). Blood samples from 44 cancer and 17 control subjects were collected. The levels of 25D did not differ between the cancer and control groups at T1, with or without controlling for covariables (both $p>0.05)$, neither did it significantly vary over time among the cancer patients $(\mathrm{p}=0.60)-\mathrm{M} \pm \mathrm{SE}=$ $28 \pm 4 \mathrm{ng} / \mathrm{ml}$ at T1, $22 \pm 4 \mathrm{ng} / \mathrm{ml}$ at T2, and $27 \pm$ $4 \mathrm{ng} / \mathrm{ml}$ at T3 controlling for covariables. The current analysis of our ongoing study failed to find a correlation between cancer and its treatment and serum levels of 25D. Analysis of the complete data set may draw different results than this incomplete set. This study will proceed to assess the relationship between 25D and incidence of fever as a surrogate marker for infectious disease. 


\section{Alexis Schuck}

\section{Potential Therapeutic Benefits of Capsaicin, EGCG and Green Tea Extract, Curcumin, and Ginger on Fibromyalgia: A Review of the Literature}

Alexis Schuck, Christina Tompkins, Volker

Neugebauer, Chwan-Li Shen

Fibromyalgia (FM) is a chronic syndrome afflicting $1 \%$ to $5 \%$ of the population and disproportionately affects females compared to males. It is a generalized pain disorder occurring at any age, usually consisting of functional somatic symptoms like fatigue, mood disorders, sleep disturbance, and pain and tenderness. In fact, FM diagnosis is principally based on widespread musculoskeletal pain present for at least three months and tenderness in at least 11 of 18 tender points. The etiopathogenesis of FM remains unclear, with genetic factors, environmental triggers, and neuromodulation all possibly contributing to the onset and course of this syndrome.

The current goals of treatment are to minimize pain symptoms and improve daily function using a multi-disciplinary approach, but the efficacy of these options is often unsatisfactory. Bioactive components may be used as adjunctive therapy in combination with current drugs, or perhaps even replace them. This review delineates the effects of commonly found bioactive compounds, including curcumin, capsaicin, ginger, and epigallocatechin gallate (EGCG) and green tea extract, on FM with an emphasis on potential mechanisms at the molecular and systemic levels.

The literature suggests these compounds exert their effects in post-synaptic neurons and the dorsal root ganglion to ultimately mitigate pain sensations. Specifically, curcumin, ginger, and EGCG inhibit the release of pro-inflammatory cytokines (TNF- $\alpha, \mathrm{IL}-1 \beta$, and IL-6) by down-regulating the NF- $\mathrm{KB}$ signaling pathway. Capsaicin desensitizes the TRPV1 channel receptor in the dorsal root ganglion to decrease pain perception in human subjects. Although encouraging results were noted in this review, further cellular, animal, and human experiments are needed to fully understand the mechanism of action for each compound and eventually improve the treatment regimen for fibromyalgia.

\section{Miriam Shayeb}

\section{The Application of Renaissance Humanism Concepts to the Development of Resilience in Medical Students}

\author{
Miriam Shayeb, Cheryl Erwin
}

Introduction: Several works of art, literature, and philosophy from the period of Renaissance Humanism highlight concepts that can serve as mechanisms of resilience in medical education. As a concept in positive psychology, resilience refers to the ability to cope with the various stressors of life. The work of Renaissance Humanists focused on individuals' ability to flourish amidst adversity, thereby exemplifying forms of resilience. The goal of this research was to review a broad range of sources by Renaissance Humanists that demonstrate how humanistic concepts can be applied as mechanisms of resilience.

Methods: EndNote software was used to create an annotated bibliography of thirty-nine primary and secondary sources from Renaissance Humanism and to categorize them based on their application to different humanistic concepts.

Findings: The Renaissance was a period of fertile humanism as well as political upheaval, plague, and crisis. Resilience was explored as a personal quality of the Renaissance thinkers. Several sources from this period exemplified how texts about science and medicine contributed to improvements in anatomical knowledge, expanded access to medical education, and demonstrated the importance of empathy in the practice of medicine. This application of knowledge combined with empathy can be useful in building resilience.

Conclusion: History does not predict the future, but it can enlighten it. The humanistic themes developed by Renaissance Humanists can enlighten our own challenging times as we live through a pandemic and as medical students are asked to adapt to changing demands and circumstances. An annotated 
bibliography of various artistic, literary, and scientific works of Renaissance Humanism was created for use in medical humanities courses and in further research. These sources exemplify concepts like practical knowledge, self-awareness, and empathy, which can serve as mechanisms to foster resilience among medical students.

\section{Shyam Sheladia}

\section{Age-Related Chronic Diseases and Alzheimer's Disease in Texas: A Hispanic Focused Study}

Shyam Sheladia, P. Hemachandra Reddy

The emergence of age-related chronic diseases in the United States has led to the direct increase of Alzheimer's disease (AD) prevalence, which ultimately contributes to the development of dementia. Age-related chronic diseases such as cardiovascular disease, high cholesterol, diabetes, and kidney disease contribute to the advancement of dementia. Furthermore, unmodifiable risk factors such as advancing age and genetics as well as modifiable risk factors such as socioeconomic status, educational attainment, exercise, and diet further contribute to the development of dementia. The purpose of our study is to determine the links between age-related chronic diseases/risk factors and cognitive decline within the Hispanic population of Texas and rural West Texas.

We collected data associated with the prevalence of $A D$ within the Hispanic population of Texas and rural West Texas. We also collected data related to the prevalence of age-related chronic diseases, unmodifiable risk factors, and modifiable risk factors which lead to the development of $A D$ in the Hispanic population.

Our analysis showed that Hispanics face the greatest burden of dementia due to the increase in the prevalence of overall population age, predisposing genetics, age-related chronic diseases, low socioeconomic status, low educational attainment, as well as poor lifestyle choices. Additionally, Hispanics living within rural West Texas face the added challenge of finding appropriate healthcare services.
Although it is difficult to provide a solution to certain factors such as socioeconomic status; steps can be taken to provide education to the Hispanic population regarding lifestyle changes that can be made in order to significantly reduce the risk of developing age- related chronic diseases which lead to the development of AD. Furthermore, a sincere effort by the Texas government and major hospital systems should be made to provide adequate healthcare resources to the counties of rural West Texas.

\section{Michelle Terry}

\section{Dynamic Cushioning: A Complex Relationship Between Obesity \& Trauma Patients Undergoing Laparotomy}

N Tully, M Terry, A Tucker, S Dhanasekara, C Ronaghan, R Richmond

Introduction: Obesity is generally associated with worse outcomes in trauma patients. However, there is conflicting evidence regarding the effect that increasing BMI has over the short and especially long term on these patients. We hypothesized that increasing $\mathrm{BMI}$ has an initial protective effect, but ultimately ends in worse outcomes. Therefore, we sought to elucidate the effect BMI has on outcomes in trauma patients undergoing exploratory laparotomy.

Methods: A Level 1 trauma center registry was queried to include all trauma patients that underwent exploratory laparotomies between June 2015-April 2020. Demographic factors along with clinical details and outcomes were examined. Baseline characteristics of 3 patient groups defined by BMI categories (i.e., normal-weight, overweight, and obese) and complications were compared. Simple linear and logistic regression analyses were performed to examine the associations between BMI and each complication.

Results: Records of 206 trauma patients who underwent exploratory laparotomies were analyzed. There was no significant difference in demographics or injury severity score (ISS) between groups (Table 1). When each complication was regressed on BMI, significant positive effects were seen for ICU 
stay, hospital length of stay (LOS) and return to OR. More granularly, a per-unit increase in BMI conferred a log-odds increase of 0.056 in return to OR. These results indicated that BMI had a direct positive association with ICU LOS, hospital LOS and return to OR.

Conclusion: Our findings show that with increasing BMI, postoperative complications increase following laparotomy for trauma. As rates of obesity increase, trauma surgeons must be prepared to anticipate plans of care from patient presentation to well beyond discharge to cope with more complex postoperative and post-hospital clinical courses.

\section{Christina Tompkins}

\section{Analgesic Effects of GSPE, Soy and Genistein, Naringin, and Omega-3 PUFA in the Treatment of Fibromyalgia and Possible Molecular Mechanisms: A Review of the Literature}

Christina Tompkins, Alexis Schuck, Volker

Neugebauer, Chwan-Li Shen

Fibromyalgia (FM), characterized by widespread pain, is a complex disorder that negatively impacts the quality of life of millions of Americans. Current treatment options for fibromyalgia are limited. Opioid analgesics can result in opioid use disorder while long-term drug use brings unwanted side effects and toxicities. The goals of treatment are to mitigate central pain amplification and minimize symptoms since no cure is available.

Recent studies have shown diet-derived bioactive compounds have anti-inflammatory, antioxidant, anti-apoptotic, and immuno- modulatory properties. This review discusses the effects of commonly consumed bioactive compounds, including grape seed extract, soy and genistein, omega-3 PUFA, and naringin on fibromyalgia with an emphasis on both possible molecular and systemic mechanisms and their likeness to current drug regimens.

After a thorough literature review, these bioactive compounds were found to significantly decrease FM-related inflammation through an analgesic manner in CNS neurons, DRGs, serum, immune cells, skeletal muscle, and mitochondria. Downregulated proinflammatory cytokine production, modulated superoxide catalase pathways, inhibited lipid peroxidation, and decreased immune cell recruitment were noted with bioactive compound supplementation in in vitro, animal, and human studies. GSPE and naringin affected monoamine modulators in a similar manner as current FM drugs. GSPE, naringin, and soy and genistein also acted similarly to current anticonvulsant drugs through inhibiting excitatory neurotransmissions.

These bioactive compounds have great potential as non-narcotic analgesic treatment options for FM as adjunctive therapy to mitigate drug tolerance and dosage. Although further research is warranted to determine their optimal bioavailability and therapeutic effect in humans, this approach would allow a more inclusive treatment regimen to patients facing healthcare barriers or high pharmaceutical drug costs.

\section{Varun Vemulapalli}

\section{Connexin Hemichannel Inhibitors Based on Aminoglycosides}

Cristina Natha, Varun Vemulapalli, Mariana Fiori, Cheng-Wei Chang, Guillermo Altenberg

Connexins are membrane proteins involved directly in cell-to-cell communication through the formation of gap-junctional channels. These channels result from the head-to-head docking of two hemichannels, one from each of two adjacent cells. Undocked hemichannels are also present at the plasma membrane where they mediate the efflux of molecules that participate in autocrine and paracrine signaling, but abnormal increase in hemichannel opening can lead to cell damage in disorders such as cardiac infarcts, strokes, deafness, cataracts, and skin diseases. For this reason, connexin hemichannels have emerged as a valid therapeutic target. Traditional hemichannel inhibitors are not ideal leads for the development of better drugs for clinical use because they are not specific and/or have toxic effects. Newer inhibitors are more selective and include connexin mimetic peptides, anti-connexin antibodies and drugs that reduce connexin expression such as antisense oligonucleotides. Re-purposed drugs and their derivatives are 
also promising because of the significant experience with their clinical use. Among these, aminoglycoside antibiotics developed in our laboratory have been identified as inhibitors of connexin hemichannels that do not inhibit gap-junctional channels. Here, we discuss connexin inhibitors with a focus on aminoglycoside antibiotics and our derivatives of kanamycin $A$ that inhibit connexin hemichannels, but do not have antibiotic effect.

\section{Haven Ward}

\section{Post-intervention Effects of Tai Chi and Qi Gong on Anxiety Scores: A Systematic Review and Meta-Analysis}

Haven Ward, Kevin Chin, Chathurika Dhanasekara, Dale Dunn, Chwan-Li Shen, Chanaka Kahathuduwa

Background: The practice of Tai-Chi and Qi Gong as mindfulness meditation exercises has been shown to have beneficial effects on psychological wellbeing. We aimed to systemically analyze the beneficial effects of exposure to at least 3-weeks of Tai Chi or Qi Gong on anxiety scores.

Methods: Peer-reviewed records examining the effects of Tai-Chi or Qi Gong on depressive or anxiety symptoms were searched using PudMed, Scopus, ProQuest, and Web-of-Science databases. Outcomes of the search were independently screened by two researchers for pre-determined eligibility criteria. Full-text articles of prospective controlled clinical trials examining the effects of exposure to $>3$ weeks of Tai Chi or Qi Gong on anxiety symptoms were identified. Studies were evaluated for the risk of bias using the methods in the Cochrane handbook. Pre- and post-intervention anxiety symptoms of the intervention and control groups were extracted. DerSimonian Liard random-effects meta-analyses were performed on the data using the meta package in $\mathrm{R}$ statistical software (version 4.0.2)

Results: Out of 1,306 studies that emerged on initial database search, 33 studies were deemed eligible for the meta-analysis. Mean exposure to Tai-Chi or Qi Gong during interventions ranged from 5-312 hours (mean $41.11 \pm 67.35$ hours). Tai-Chi interventions were observed to significantly decrease standardized anxiety scores vs. controls (Cohen's $d=-0.52$, [-0.82; -0.22]). Similarly, Qi Gong interventions significantly decreased standardized anxiety scores vs. controls (Cohen's $d=-0.49[-0.77 ;-0.20]$ ).

Discussion: Mind body interventions such as TaiChi and Qi Gong seems to have a moderate effect in alleviating anxiety. Our findings should inform the clinicians to encourage the use of these mind-body interventions to reduce clinical as well as subclinical anxiety. Future meta-regression analyses should examine the differential effects of mind-body interventions based on socioeconomic factors and symptom severity.

\section{Adam Wynn}

\section{Effects of Consumption of Coconut Oil or Coconut on Glycemic Control and Insulin Sensitivity: A Systematic Review and Meta-Analysis of Interventional Trials}

Adam Wynn, Amber Nelson, Megan Spradley, Chathurika Dhanasekara, Christina Robohm-Leavitt, Chwan-Li Shen, Chanaka Kahathuduwa

Background: While the often-purported claim that coconut fat is beneficial for cardiovascular health was disputed in a recent comprehensive metaanalysis, evidence on the effects of coconut fat consumption on glycemic control remains equivocal. We conducted a systematic review and meta-analysis to determine the effects of dietary coconut fats on markers of glycemic control.

Methods: Specific, predefined keyword combinations were used to search PubMed, Scopus, ProQuest, and Web-of-Science studies examining coconut derivatives' effects on glycemic control. Records were screened by three independent reviewers using pre-determined eligibility criteria. Data from randomized controlled trials (RCTs) examining acute and long-term (i.e. > 10 days) effects of coconut fat consumption on markers of glycemic control were extracted. DerSimonian Liard random effects meta-analyses were conducted using meta package in $\mathrm{R}(4.0 .2)$. 
Results: 14 RCTs on acute effects and 4 RCTs on long-term effects of coconut oil were included. Meals containing coconut fat significantly increased incremental AUC of glucose $(\Delta=162.48 \mathrm{mg} \times \mathrm{min} / \mathrm{dL}$ $[2.74,322.22], p=0.046)$ and significantly decreased incremental AUC of insulin vs. control meals $(\Delta=$ $-459.13 \mathrm{mlU} \times \mathrm{min} / \mathrm{L}[-889.98,-28.27], \mathrm{p}=0.037)$. Furthermore, coconut fat-containing meals resulted in a small yet significant increase in fasting blood glucose vs. control meals $(\Delta=2.73 \mathrm{mg} / \mathrm{dL}[95 \% \mathrm{Cl}$ : $0.50,4.95], p=0.0162)$. Meta-analyses of studies examining long-term effects of coconut fat consumption did not show significant differences in fasting insulin, HOMA- $\beta$, or HOMA-IR between coconut fat vs. control groups.

Conclusions: Dietary coconut fat seems to be associated with a decreased post-prandial insulin response, possibly causing a minimal, yet increased post-prandial glycemic response. Coconut oil does not seem to have long-term benefits for glycemic control. These results disprove the popular claims that coconut fat improves glycemic control. 